\title{
Total pancreatectomy for cholangiocarcinoma of the distal common bile duct associated with lipomatous pseudohypertrophy of pancreas
}

\author{
Subani Priyangika Jayatunge, Gayana Mahendra, Sajith Siyabalapitiya, \\ Rohan Chaminda Siriwardana, Chandika Liyanage
}

\begin{abstract}
Introduction: Fatty replacement of the exocrine pancreas also known as lipomatosis, adipose atrophy or lipomatous pseudohypertrophy is a well recognize benign condition. This fatty infiltration can occur either focally or involving the whole pancreas. Case Report: We report a rare case of lipomatous pseudohypertrophy of the pancreas associated with cholangiocarcinoma of the distal common bile duct (CBD). A 51-yearold female presented to our unit with a history of obstructive jaundice. Radiological imaging suggested a cholangiocarcinoma of the distal CBD with pancreatic lipomatosis, without any clinical evidence of exocrine or endocrine dysfunction. She underwent a radical bile duct excision and a total pancreatectomy. Histology revealed lipomatosis of the pancreatic body and tail with sparse pancreatic tissue adjacent to the tumor. After an uneventful recovery, she was
\end{abstract}

Subani Priyangika Jayatunge ${ }^{1}$, Gayana Mahendra², Sajith Siyabalapitiya ${ }^{3}$, Rohan Chaminda Siriwardana ${ }^{4}$, Chandika Liyanage $^{4}$

Affiliations: ${ }^{1} \mathrm{MBBS}$, Trainee, Hepato-Pancreatico-Biliary Unit, North Colombo Teaching Hospital, Ragama, Sri Lanka; ${ }^{2} \mathrm{MBBS}$, Dip.Path, MD Hist.Path, Consultant Histopathologist, Department of Pathology, Faculty of Medicine, Ragama, Sri Lanka; ${ }^{3} \mathrm{MBBS}, \mathrm{MD}, \mathrm{MRCP}$, Counsultant Endocrinologist, North Colombo Teaching Hospital, Ragama, Sri Lanka; ${ }^{4}$ MBBS, MS, MRCS, Consultant GI and Hepato-Pancreatico-Biliary Surgeon, North Colombo Teaching Hospital, Ragama, Sri Lanka.

Corresponding Author: Dona Subani Priyangika Jayatunge, 19/2 Rajagaha Mawatha, Kanuwana, Kurunduwaththa, Ja-ela, Sri Lanka; Ph: 00940774612926 , Fax: 0094 0112958337; Email: dspjayatunge@gmail.com

Received: 17 February 2015

Accepted: 25 March 2015

Published: 14 April 2015 started on lifelong insulin and pancreatic enzyme supplements. Even though pathogenesis of pancreatic lipomatosis remains controversial, it is most likely due to obstruction of the pancreatic duct in this presentation. Conclusion: It is import to undertake total pancreatectomy for this dual presentation with specialized endocrinological support in the stormy postoperative period.

Keywords: Cholangiocarcinoma, Lipomatous pseudohypertrophy of the pancreas, Total pancreatectomy

\section{How to cite this article}

Jayatunge SP, Mahendra G, Siyabalapitiya SS, Siriwardana RC, Liyanage C. Total pancreatectomy for cholangiocarcinoma of the distal common bile duct associated with lipomatous pseudohypertrophy of pancreas. Int $J$ Hepatobiliary Pancreat Dis 2015;5:30-34.

Article ID: 100031IJHPDSJ2015

$* * * * * * * * *$

doi:10.5348/ijhpd-2015-31-CR-6

\section{INTRODUCTION}

Fatty replacement of the exocrine pancreas also known as lipomatosis, adipose atrophy or lipomatous pseudohypertrophy is a well recognize benign condition [1]. This fatty infiltration can occur either focally or involving the whole pancreas. Focal fatty infiltration is commonly seen in the obese and elderly and it is the most common degenerative lesion involving the pancreas [2]. 


\section{CASE REPORT}

A 51-year-old female presented to the hepatobiliary department of the North Colombo Teaching Hospital with a one-month history of intermittent fever, epigastric pain and features suggestive of obstructive jaundice. Liver biochemistry confirmed obstructive jaundice. (Bilirubin: Total 16, $8 \mathrm{mg} / \mathrm{dl}$; direct $7.6 \mathrm{mg} / \mathrm{dl}$, alkaline Phosphatase (ALP) 4772IU/l, AST 235 IU/l, ALT 155 IU/1.) with elevated Carbohydrate antigen 19.9 ( CA19.9) of $322,630 \mathrm{IU} / \mathrm{ml}$ ).

Abdominal ultrasonography, computed tomography $(\mathrm{CT})$ and magnetic resonance imaging (MRI) revealed markedly dilated intrahepatic bile ducts and common bile duct (CBD diameter $2 \mathrm{~cm}$ ), associated with narrowing of the distal end of the common bile duct with thickening suggestive of distal cholangiocarcinoma. Her main pancreatic duct was dilated but the CT scan could not visualize any pancreatic tissue (Figure 1). With the above investigations she was diagnosed of having a distal CBD cholangiocarcinoma associated with pancreatic lipomatosis.

The patient had no significant comorbidities including diabetes and had no features of chronic malabsorption, suggestive of endocrine or exocrine pancreatic dysfunction.

After discussion at the hepato-pancreato-biliary (HPB) multidisciplinary meeting, she underwent a radical bile duct excision and a total pancreatectomy. At surgery the pancreas could not be visualized and was found to have been replaced with fatty tissue. During the post operative period she was managed in the high dependency unit with stringent blood sugar control with insulin infusion and pancreatic enzyme supplements.

The specimen included the gallbladder, complete extra-hepatic biliary tree, 14 lymph nodes, duodenum and the fatty tissue in the pancreatic bed (Figure 2). The histological analysis of the specimen confirmed adenocarcinoma of the distal CBD, predominantly of biliary type with foci of gastric foveolar type and high grade biliary intraepithelial neoplasia in the common bile duct. The tumor infiltrated the pancreas, duodenum and retro-peritoneal fatty tissue with vascular and perineural invasion. Six out of the fourteen regional lymph nodes showed tumor metastasis giving the tumor stage as pT3NIMx. The histology of the pancreas revealed mature fatty tissue containing aggregates of pancreatic endocrine cells with prominent neural bundles. Almost the total pancreas was replaced by fat except for a few areas in the region of the head. The exocrine elements were completely absent (Figure 3). There was no evidence of a neoplasm of the pancreas and features were compatible with diffuse involved pancreatic lipomatosis of the body and tail (Figure 4).

The patients had an uneventful recovery and was discharged on post operative day 14 with a management plan by the endocrinologist which included 12 units of mixtard insulin in the morning, 6 units of soluble insulin

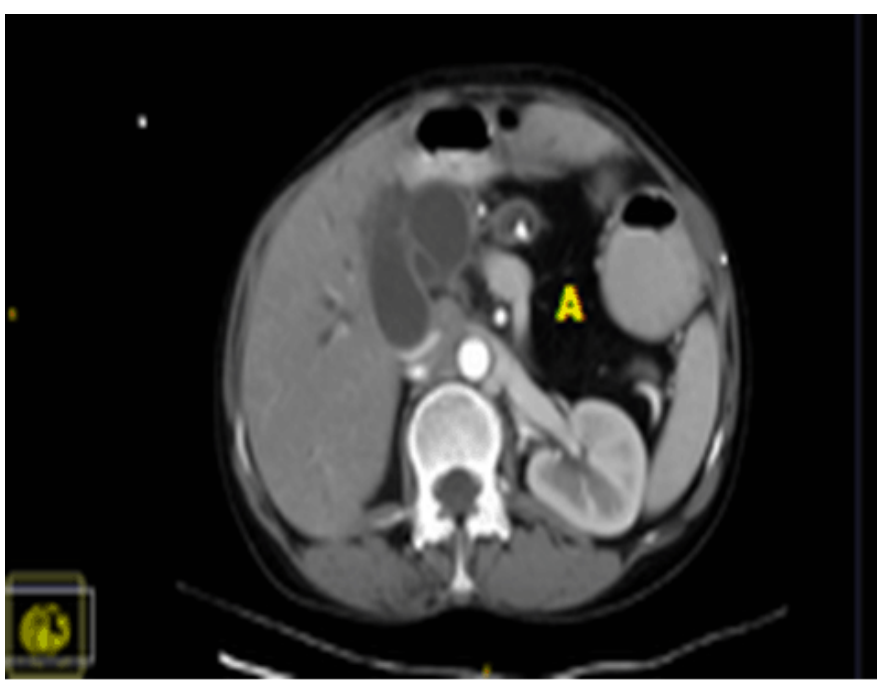

Figure 1: Computed Tomography in this patient reveals dilated pancreatic duct containing multiple calculi with atrophy of pancreatic tissue (marked A).

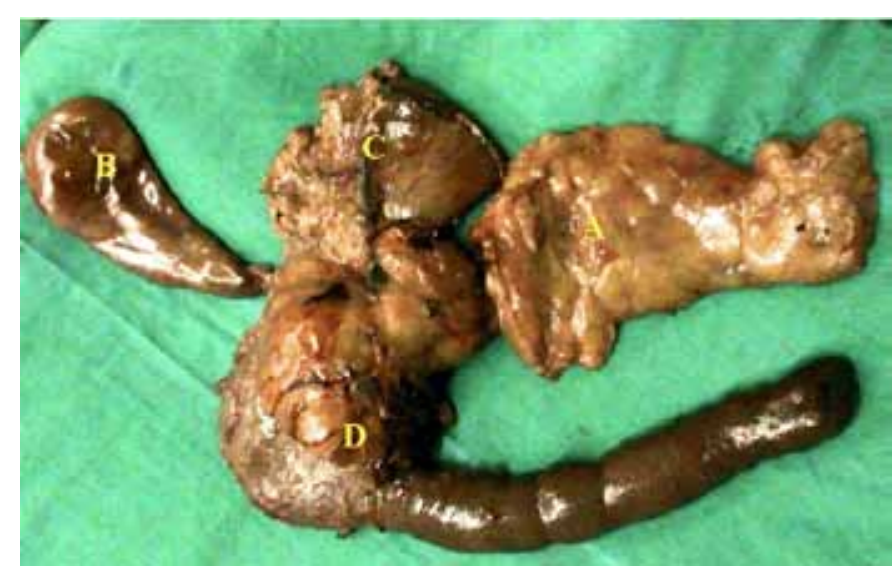

Figure 2: Image of the resected surgical specimen (A: Lipomatosis pancreas, B: gallbladder, C: distal stomach , D: Duodenum).

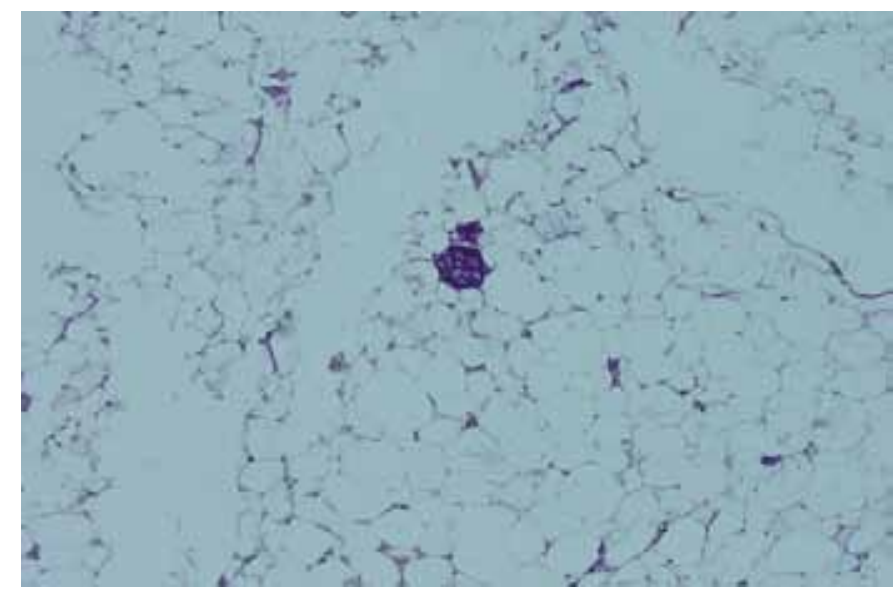

Figure 3: Microscopic image of body of the pancreas demonstrating clusters of endocrine cells in a fatty background. There is no exocrine element of the pancreas identified. 


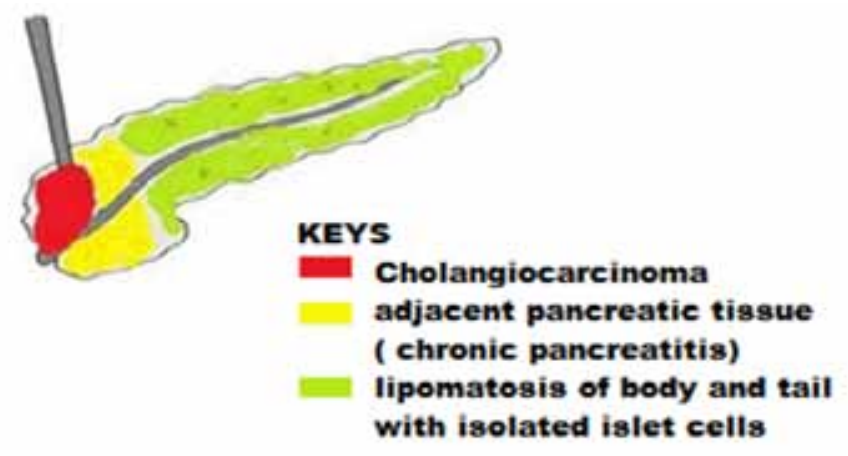

Figure 4: Diagram of the pancreas illustrating the macroscopic the distribution of pancreatic lipomatosis

at evening and pancreatic enzyme supplementation. She was started on adjuvant gemcitabine based chemotherapy once her blood sugar control stabilized. After one year following surgery patient has successfully completed her six months of chemotherapy and free of tumour recurrence.

\section{DISCUSSION}

Diffuse lipomatosis or lipomatous pseudohypertrophy (LiPH) of the pancreas is a rare presentation with an uncertain aetiology. LiPH of the pancreas was first described by Hantelmann in 1931 [3]. It is characterize by

1. Increase in the size and weight of pancreas

2. Almost complete absence of pancreatic exocrine tissue due to pathological replacement with adipose tissue

3. Preservation of the system of duct and islets of Langerhans [3, 4, 5].

Among several cases reported of LiPH of pancreas, the sites of involvement of lipomatosis were the entire pancreas, body and pancreatic tail, pancreatic head and uncinate. The involvement of the entire pancreas was most commonly observed [5].

This disease entity is reported to be associated with viral infections [3], chronic hepatic disease [3], congenital abnormalities such as Shwachman-Diamond syndrome and cystic fibrosis [6]. Diabetes mellitus, steroid therapy, Cushing's syndrome, hemochromatosis, hereditary and chronic pancreatitis have also been reported to be related to diffuse fatty replacement of pancreas [6, 7]. Though the mechanism is controversial, obstruction of the main pancreatic duct may also lead to fatty replacement of the distal portion of the pancreas [8]. The stages of pancreatic lipomatosis have been described as diffuse type, headnodular type and tail-nodular type.

The clinical symptoms of pancreatic lipomatosis depend on the degree of fatty infiltration of the pancreas [6]. Patients with small focal fatty infiltrations are usually asymptomatic, however with extensive pancreatic lipomatosis the patients may present with atypical abdominal pain, malabsorption and diabetes mellitus due to exocrine and endocrine dysfunction $[1,6,7]$.

Pancreatic lipomatosis is visible on abdominal sonography, CT and MRI. An increase in the echogenicity of the pancreas is noted on abdominal sonography and the CT shows more specific fat density in the pancreas. In both short and long TR images MRI reveals a variable sized pancreas with signal intensity [7].

In this patient the aetiology was most likely related to obstruction of the main pancreatic duct in chronic pancreatitis causing lipomatosis. She was found to have diffuse pancreatic lipomatosis of the pancreatic body and tail with few spared areas in the head. She had satisfactory compensatory function from the tissues in the pancreatic head and the scattered endocrine elements in the fat tissue in lipomatosis pancreas.

As she had a distal cholangiocarcinoma she required a Whipple procedure, and due to the absence of the pancreas a "total pancreatectomy" had to be performed to include all areas of the pancreas. This was because there was no pancreas tissue for reconstruction.

\section{CONCLUSION}

It is extremely rare that distal cholangiocarcinoma is associated with pancreatic lipomatous pseudohypertrophy. We report this to enlighten the readership of radiological and histological diagnosis of pancreatic LiPH and to highlight the need of a total pancreatectomy for this combination. It is important to undertake this procedure at a specialized centre with support from an endocrinologist to manage the stormy postoperative period. Since it will be a lifelong replacement for patient, it is important to educate the patient of the life style change and evaluate if it is a suitable procedure for the particular individual.

$$
* * * * * * * * *
$$

\section{Author Contributions}

Subani Priyangika Jayatunge - Substantial contributions to conception and design, Drafting the article, Final approval of the version to be published

Gayana Mahendra - Substantial contributions to conception and design, Drafting the article, Revising it critically for important intellectual content, Final approval of the version to be published

Sajith Siyabalapitiya - Substantial contributions to conception and design, Drafting the article, Revising it critically for important intellectual content, Final approval of the version to be published

Rohan Chaminda Siriwardana-Substantial contributions to conception and design, Drafting the article, Revising it critically for important intellectual content, Final approval of the version to be published

Chandika Liyanage - Substantial contributions to conception and design, Drafting the article, Revising 
it critically for important intellectual content, Final approval of the version to be published

\section{Guarantor}

The corresponding author is the guarantor of submission.

\section{Conflict of Interest}

Authors declare no conflict of interest.

\section{Copyright}

(C) 2015 Subani Priyangika Jayatunge et al. This article is distributed under the terms of Creative Commons Attribution License which permits unrestricted use, distribution and reproduction in any medium provided the original author(s) and original publisher are properly credited. Please see the copyright policy on the journal website for more information.

\section{REFERENCES}

1. Anand R, Narula MK, Chaudhary V, Agrawal R. Total pancreatic lipomatosis with malabsorption syndrome. Indian J Endocrinol Metab 2011 Jan;15(1):51-3.
2. Patel S, Bellon EM, Haaga J, Park CH. Fat replacement of the exocrine pancreas. AJR Am J Roentgenol 1980 Oct;135(4):843-5.

3. Yasuda $M$, Niina $Y$, Uchida $M$, Fujimori $N$, Nakamura T, Oono T, et al. A case of lipomatous pseudohypertrophy of the pancreas diagnosed by typical imaging. JOP $2010 \mathrm{Jul}$ 5;11(4):385-8.

4. Shimada M, Shibahara K, Kitamura H, Demura Y, Hada M, Takehara A, et al. Lipomatous Pseudohypertrophy of the Pancreas Taking the Form of Huge Massive Lesion of the Pancreatic Head. Case Rep Gastroenterol 2010 Oct 22;4(3):457-64.

5. Izumi S, Nakamura S, Tokumo M, Mano S. A minute pancreatic ductal adenocarcinoma with lipomatous pseudohypertrophy of the pancreas. JOP 2011 Sep 9;12(5):464-8.

6. Sodhi KS, Vyas S, Khandelwa N, Gupta R. Complete pancreatic lipomatosis. Indian J Gastroenterol 2010 Mar;29(2):65.

7. Lin WC1, Chen JH, Lin CH, Shen WC. Rapidly progressive pancreatic lipomatosis in a young adult patient with transfusion-dependent myelodysplastic syndrome. J Formos Med Assoc 2007 Aug;106(8):676-9.

8. Toyama N, Kamiyama H, Suminaga Y, Namai K, Ota M, Konishi F. Pancreas head carcinoma with total fat replacement of the dorsal exocrine pancreas. J Gastroenterol 2004 Jan;39(1):76-80.

\section{ABOUT THE AUTHORS}

Article citation: Jayatunge SP, Mahendra G, Siyabalapitiya SS, Siriwardana RC, Liyanage C. Total pancreatectomy for cholangiocarcinoma of the distal common bile duct associated with lipomatous pseudohypertrophy of pancreas. Int J Hepatobiliary Pancreat Dis 2015;5:30-34.

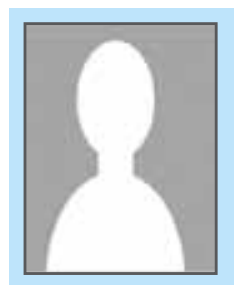

Subani Jayatunge is a Trainee at Department of Surgery, Faculty of medicine, University of Kelaniya, Ragama, Sri Lanka. She earned the undergraduate degree (MBBS) from Faculty of Medicine, University of Kelaniya, Ragama, Sri Lanka in 2012. Her research interests include Hepatobiliary surgery. E-mail: dspjayatunge@gmail.com

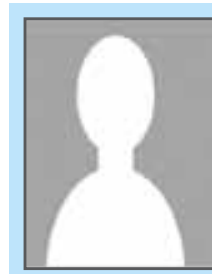

Gayana Mahendra is Senior Lecturer at Department of Pathology, Faculty of Medicine, University of Kelaniya, Sri Lanka. She earned the undergraduate degree from Faculty of Medicine, University of Colombo, Sri Lanka and postgraduate degrees form Post Graduate Institute of Medicine, University of Colombo, Sri Lanka. She has published research papers in national and international academic journals. Her research interests include soft tissue and bone pathology and gastrointestinal pathology E-mail: baggmahendra@yahoo.co.uk

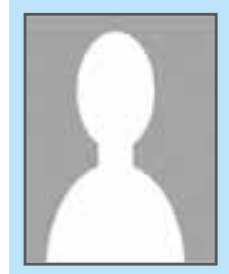

Sajith Siyabalapitiya is Consultant Endocrinologist at North Colombo Teaching Hospital, Ragama, Sri Lanka. He has published research papers in national and international academic journals. 


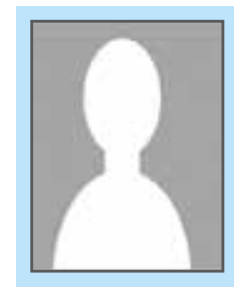

Rohan Chaminda Siriwardana is a Senior Lecturer, Consultant GI, HPB and Liver Transplant Surgeon at Department of Surgery, Faculty of Medicine, University of Kelaniya, Ragama, Sri Lanka. He has published many research papers in national and international academic journals and his research interests include hepatobiliary and liver transplant surgery.

E-mail: rohansiriwardana@yahoo.com

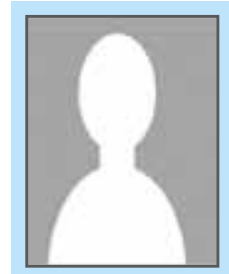

Chandika Liyange is a Senior Lecturer at Department of Surgery, Faculty of medicine, University of Kelaniya, Ragama, Sri Lanka.

Access full text article on other devices

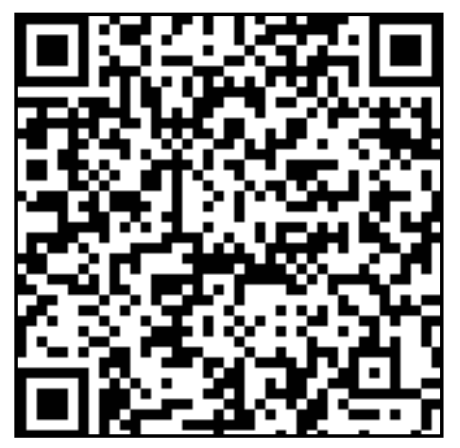

Access PDF of article on other devices

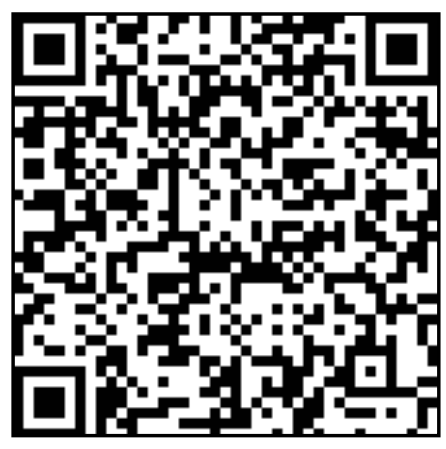

